0038-1098(95)00597-8

\title{
EFFECTS OF A TIME-DEPENDENT TRANSVERSE ELECTRIC FIELD ON THE QUANTUM TRANSPORT IN NARROW CHANNELS
}

\author{
C. S. Chu and C. S. Tang \\ Department of Electrophysics, National Chiao Tung University, \\ Hsinchu 30050, Taiwan, R.O.C. \\ (Received and accepted 28 August 1995 by A.H. MacDonald)
}

\begin{abstract}
The quantum transport in a narrow channel $(\mathrm{NC})$ is studied in the presence of a timedependent delta-profile electric field. The electric field is taken to be transversely polarized, with frequency $\omega$, causing inter-subband and inter-sideband transitions. Suppression in the dc conductance $G$ is found, which escalates with the chemical potential. There are structures in $G$ which are related to the quasi-bound state (QBS) features. Major dip, and dip-and-peak, structures occur when an incident electron makes transition to a subband edge by absorbing or emitting one, and two, $\hbar \omega$, respectively. Structures associated with $3 \hbar \omega$ processes are recognized. The QBS are closely associated with the singular density of states (DOS) at subband bottoms. Our results demonstrate that, due to this singular features of the DOS, the interaction of the electron with the electric field has to be treated beyond finite order perturbation.
\end{abstract}

Keywords: A. nanostructures, A. semiconductors, D. electronic transport, D. radiation effects

Quantum transport in NC has received a lot of attcntion in recent years. These channels can be realized experimentally within a split-gate configuration $[1,2]$. The channels connect adiabatically on each side to a twodimensional clectron gas. Energy levels of the channels are quantized into one-dimensional subbands which give rise, in the ballistic regimc, to a quantized $G[1,2]$. The singular feature of the DOS near a subband edge leads to impurity-induced dip structures in $G$ when the scatterer is attractive [3-9]. These dip structures are associated with the formation of impurity-induced QBS [1].

More recently, there are growing interest in the timedependent phenomena in quantum point contact (QPC) systems [10 15]. Firstly, the optical absorption coefficient of a QPC has been calculated [10], up to secondorder in the electron-photon coupling, and a proposal is made that the optical absorption in the QPC can be used to characterize the lateral confining potential of the
QPC. Secondly, the photon-assisted quantum transport in QPC systems $[11-15]$ has been studied while ministeps are predicted to appear in $G$ versus $\mu$ [12].

On the other hand, QBS features are found in $G$ when a point barrier oscillates in a narrow channel [16]. These features, that the dc conductance $G$ exhibits dip, or peak structures when the chemical potential $\mu$ is at $n \hbar \omega$ above the threshold energy of a subband, persists even in the case of a finite-range oscillating barrier, including the case when the barrier range $a \gg \lambda_{F}[17]$. The oscillating barrier is uniform in the transverse direction and does not induce inter-subband transitions. However, the presence of the oscillating barrier, as long as it has a longitudinal profile, breaks the longitudinal translational invariance. Thus the electrons are relieved from conserving the longitudinal momentum, making the inter-sideband transitions possible. The sideband index $n$ labels those electrons which net energy change. 
is $n \hbar \omega$, as a result of interacting with the oscillating barrier. The physical origin of these QBS, as pointed out by Bagwell and Lake [16] to be essentially similar to that in the impurity-induced QBS, is associated with the singular DOS at subband bottoms [18]. From the above understanding, the QBS features are induced by an oscillating barrier with a longitudinal profile. Hence it is intcresting to ask whether a transversely polarized time-dependent electric field, with a longitudinal profile, could induce similar, or richer, QBS features?

In this paper, we study the quantum transport in a NC which is acted upon by an electric field. The transverse confinement of the NC is modelled by a quadratic potential [19] and the time-dependent transverse electric field $\vec{E}(\vec{x}, t)=E(x) \cos (\omega t) \hat{y}$ is taken to have a deltaprofile. The effect of this electric field is represented by a potential $e E_{0} y \delta(x) \cos (\omega t)$, where $-e$ is the charge of an electron and $E_{0}$ is essentially the integral of $E(x)$ with respect to the longitudinal coordinate $x$. Even though our delta-type profile electric field does not correspond to the experimental situation, we still expect that features obtained in this calculation persist in the more realistic case of a finite-range-profile electric field. The reasons being, firstly, that this model has incorporated the essential processes: the inter-subband, the intra-subband, and the inter-sideband transitions. Secondly, our approach has a renormalization feature which is important in narrow channels, especially when QBS should exist [9]. Thirdly, as discussed above, our recent study [17] shows that the features of the QBS found in the point-oscillating-barrier [16] persist even when the oscillating barrier has a large-finite-range profile.

More importantly, our results demonstrates that the delta profile does not introduce artifacts that mask the features in a large-finite-range profile. [17] Thus the key, and the most significant, role of the delta profile is to break the conservation of the longitudinal momentum, allowing the electron to undertake an energy change of $n \hbar \omega$, other than the subband energy difference. Even though the potential is not the same as the delta-profile electric field in this paper, the results [17] hint that the delta-profile assumption is a reasonable simplification to the more realistic case of a finite-profile electric field. The results thus obtained should be qualitatively sound, at least for a first attempt to this complicated problem. We intend to extend, in the near future, our calculation to the case of a finite-profile electric field.
Choosing the exergy unit $E^{*}=\hbar^{2} k_{F}^{2} / 2 m^{*}$, the length unit $a^{*}=1 / k_{F}$, the time unit $t^{*}=\hbar / E^{*}$, and $E_{0}$ in units of $E^{*} / e$, the dimensionless two-dimensional Schrödinger equation becomes

$$
\left[-\nabla^{2}+\omega_{y}^{2} y^{2}+E_{0} y \delta(x) \cos (\omega t)\right] \Psi(\vec{x}, t)=i \frac{\partial}{\partial t} \Psi(\vec{x}, t) .
$$

Here $k_{F}$ is a typical Fermi wavevector of the reservoir. The transverse energy levels are quantized, given by $\varepsilon_{n}=(2 n+1) \omega_{y}$. For a $n$th subband electron incident along $\hat{x}$, and with energy $\mu$, the scattering wavefunction is in the form

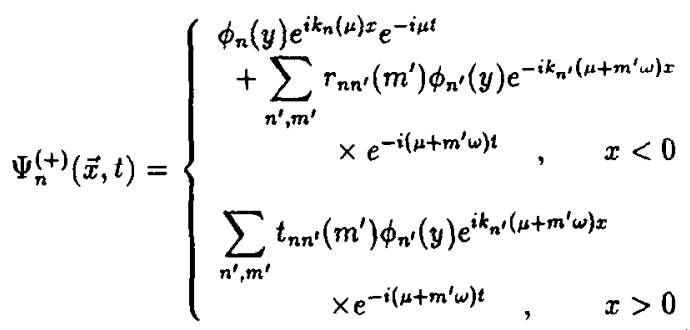

where $n^{\prime}$ and $m^{\prime}$ are the final subband and sideband indices, respectively. A positive (negative) sideband index $m^{\prime}$ corresponds to the case when the reflected or the transmitted wave has absorbed (emitted) a net energy of $m^{\prime} \hbar \omega$. The wavevector $k_{n}(\mu)=\sqrt{\mu-(2 n+1) \omega_{\nu}}$ is the effective wavevector for the electron with energy $\mu$ and in the $n$th subband.

Matching the wavefunctions at $x=0$ and given the expression of the matrix element

$$
\left(l|y| n^{\prime}\right\rangle=\frac{1}{\sqrt{2 \omega_{y}}}\left[\sqrt{n^{\prime}} \delta_{l, n^{\prime}-1}+\sqrt{n^{\prime}+1} \delta_{l, n^{\prime}+1}\right],
$$

we obtain the equations for the reflection coefficients $r_{n l}(m)$ and the transmission coefficients $t_{n l}(m)$,

$$
t_{n l}(m)-r_{n l}(m)=\delta_{m, 0} \delta_{n, l},
$$

and

$$
\begin{aligned}
k_{l}(\mu+m \omega) t_{n l}(m)= & \frac{E_{0}}{4 i} \sum_{n^{\prime}, m^{\prime}}\left[\delta_{m^{\prime}, m+1}+\delta_{m^{\prime}, m-1}\right] \\
& \times\left\langle l|y| n^{\prime}\right\rangle t_{n n^{\prime}}\left(m^{\prime}\right) \\
& +\delta_{m, 0} \delta_{n, l} k_{n}(\mu) .
\end{aligned}
$$

From Eq. (3) and the expression of $k_{n}(\mu)$, it is clear that the electric-field-induced electron transitions do not conserve the longitudinal momentum. These transition processes are made possible as long as the electric field has a longitudinal profile, be it delta-like or large finiterange-like. 
To show the contribution to $G$ from various inelastic processes, we write $G=\left(2 e^{2} / h\right) \sum_{n=0}^{N} G_{n}$, where $N+1$ is the number of propagating incident subbands. The contribution to $G$ from the $n$th subband incident electrons is $G_{n}=\sum_{n^{\prime}=0}^{N} \sum_{m^{\prime}} G_{n n^{\prime}}^{m^{\prime}}$, where $G_{n n^{\prime}}^{m^{\prime}}$ is related to the transmission coefficients, given by

$$
G_{n n^{\prime}}^{m^{\prime}}=\frac{k_{n^{\prime}}\left(\mu+m^{\prime} \omega\right)}{k_{n}(\mu)}\left|t_{n n^{\prime}}\left(m^{\prime}\right)\right|^{2}
$$

The conservation of the dc current, given by the condition $G_{n}=\operatorname{Re}\left[t_{n n}(0)\right]$, is used to check our results.

In our numerical examples, the $\mathrm{NC}$ is taken to be that in a high mobility $\mathrm{GaAs}-\mathrm{Al}_{x} \mathrm{Ga}_{1-x}$ As with a typical electron density $n \sim 2.5 \times 10^{11} \mathrm{~cm}^{-2}, m^{*}=0.067 \mathrm{~m}_{e}$, and $\lambda_{F}=500 \AA$. Correspondingly, our choice of energy unit $E^{*}=\hbar^{2} k_{F}^{2} / 2 m^{*}=9 \mathrm{meV}$, length unit $a^{*}=1 / k_{F}=$ $79.6 \AA$, and frequency unit $\omega^{*}=E^{*} / \hbar=13.6 \mathrm{THz}$. We also take $\omega_{y}=0.035$, such that the effective channel width is of the order of $10^{3} \AA$, and $E_{0}=0.035$.

In Fig. $1, G$ is plotted as a function of $X$, instead

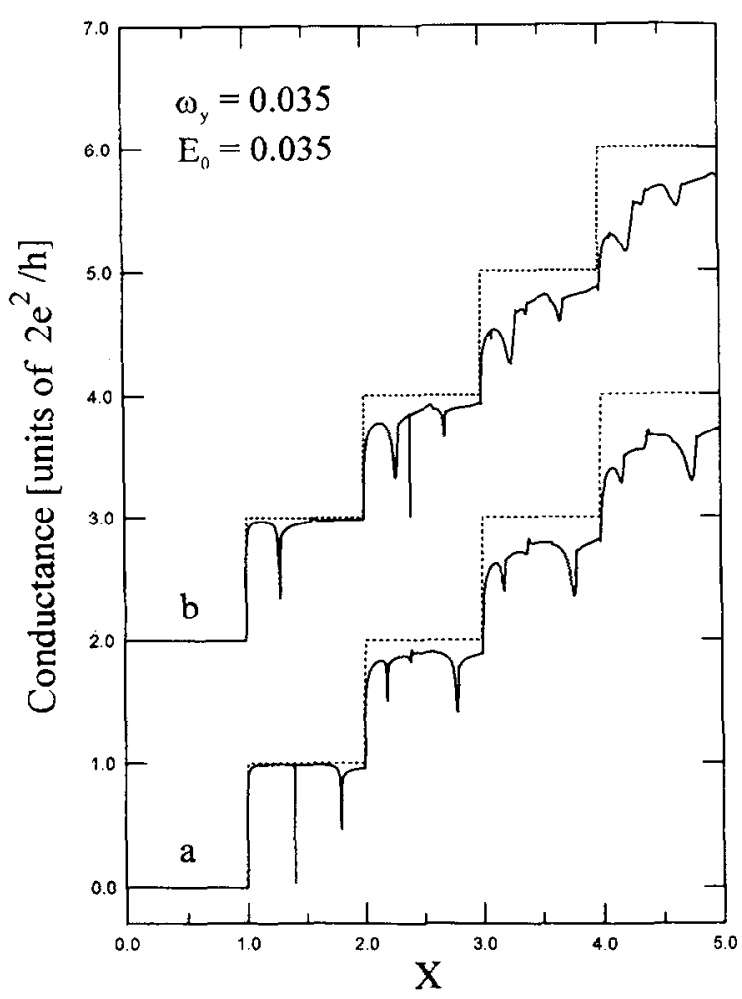

FIG. 1. Conductance $G$ as a function of $X . E_{0}=$ 0.0 for the dotted curves and $E_{0}=0.035$ for the solid curves. The frequencies are: " $a$ " $\omega=0.014$, and "b" $\omega=0.049$. Curve " $b$ " is vertically offset for clarity. of $\mu$, where $X=\left[\left(\mu / \omega_{y}\right)+1\right] / 2$ is the number of propagating channel. The dotted curves are the unperturbed results. The solid curves in Figs. 1(a), and 1(b) have different electric field frequencies: $\omega=0.014$, and 0.049 , which correspond to $190 \mathrm{GHz}$, and $666 \mathrm{GHz}$, respectively. The energy interval $\hbar \omega$ corresponds to an interval $\Delta X=\omega /\left(2 \omega_{y}\right)$ on the ordinate. Thus $\Delta X=0.2,0.7$, respectively, in Figs. 1(a), and (b). The two figures show a suppression in $G$, which increases with $X$. There are dip structures, dip-and-peak structures in $G$ and the structures are sharper in the smaller $\omega$ region. The dip structures around $X=1.8,2.8,3.8$, and 4.8 in Fig. 1 (a) and around $X=1.3,2.3,3.3$, and 4.3 in Fig. 1(b), correspond to the situations when $X+\Delta X=N+1$ are in the neighbourhood of the $N$ th subband edge. At these values of $X$, the electron can absorb one $\hbar \omega$ and makes an inter-subband transition to the band bottom of the $N$ th subband which DOS, being proportional to $\sum_{n=0}^{N} 1 / k_{n}(\mu+\omega)$, is singular because of the vanishing $k_{N}(\mu+\omega)$.

It is unexpected, though not entirely surprising, that $G$ exhibits such a dip-like drop, rather than a spike-like peak, in spite of the opening up of a new electric-fieldassisted transmission channel. This can be understood in light of the formation of a QBS $[4,16]$. The QBS, formed at energy near a subband edge, traps temporarily a conduction electron and gives rise to a drop in $G$. However, due to the interaction of the electron with the electric field, the trapped electron can be excited back out of the QBS, resulting in a smaller $G$ reduction: $|\Delta G|<1$, in units of $2 e^{2} / h$. In contrast, the impurityinduced dips are the results of merely elastic scattering and thus have $G$ reduction $|\Delta G|=1[3,4]$. Our results demonstrate the manifestation of a new, and electricfield-induced, QBS formed from both inter-subband and inter-sideband transitions.

Other dip structures around $X=2.2,3.2$, and 4.2 in Fig. 1(a), and around $X=2.7,3.7$, and 4.7 in Fig. 1(b) correspond to the situation $X-\Delta X=N+1$ when the electron can emit one $\hbar \omega$ and makes an inter-subband transition to the $N$ th subband edge. Again, the causes of these structures are the QBS. Structures other than one $\hbar \omega$ processes can also be identified. The structures around $X=1.4,2.4,3.4$, and 4.4 in Fig. $1(\mathrm{a})$, and around $X=2.4,3.4$, and 4.4 in Fig. 1(b) correspond to the situation $X-2 \Delta X=N+1$ when the electron can emit $2 \hbar \omega$ and makes a transition to the $N$ th sub- 
band edge. We remark, however, that the transitions are intra-subband transitions and that the structures, besides the first dip, do not carry a simple dip-profile. Rather, they are dip-and-peak structures which betray the competition between the trapping of electrons by the QBS and the opening up of a new electric-field-assisted transmission channel. The structures in $\mathrm{G}$ due to $3 \hbar \mathrm{w}$ processes are at $X=2.6,3.6$, and 4.6 in Fig. 1 (a) and at $X=3.1$, and 4.1 in Fig. 1(b). These structures are more pronounced for larger $\omega$. Overall, as far as the dependence on $X$ is concerned, the suppression in $G$ increases as $X$ is increasing. In addition, the dip structures and the dip-and-peak structures are sharper in the smaller $X$ region.

In the above calculation, for practical purposes, we have to impose a cutoff to the maximum number of net $\hbar \omega$ involved in the electron transmission and the accu racy of our results is checked by the conservation of current condition, given after Eq. (6). For our chosen values for $E_{0}$, it is found that processes involve more than three net $\hbar \omega$ are essentially negligible. The $m$ net $\hbar \omega$ processes we refer to are the processes that the emanating electrons have absorbed or emitted $m \hbar \omega$. There is, of course, no restriction to the number of interactions occurring during the transmission of the electron. This feature is contained in our results: that we have incorporated $E_{0}$ and $\omega$ to all orders even for a finite cutoff to the maximum number of net $\hbar \omega$ processes. Thus $G$ is found to converge rapidly with the maximum number of net $\hbar \omega$ processes included. This is effectively a renormalization procedure which is deemed necessary due to the singular DOS near a subband edge.

In conclusion, the QBS features are found in a timedependent transverse clectric field with a delta profile. It has been argued that our results have qualitative implications to the case of a finite-profile electric field, and hence the photon effects. Further study, however, is needed to reach the final conclusion.

Acknowledgement - This work was partially supported by the National Science Council of the Republic of China through Contract No. NSC83-0208-M-009-060.

\section{References}

1. B. J. van Wees, H. van Houton, C. W. J. Beenakker, J. G. Williamson, L. P. Kouwenhoven, D. van der Marel, and C. T. Foxon, Phys. Rev. Lett. 60, 848 (1988).

2. D. A. Wharam, T. J. Thornton, R. Newbury, M. Pepper, H. Ahmed, J. E. F. Frost, D. G. Hasko, D. C. Peacock, D. A. Ritchie, and G. A. C. Jones, J. Phys. C 21, L209 (1988).

3. C. S. Chu, and R. S. Sorbello, Phys. Rev. B 40, 5941 (1989).

4. P. Bagwell, Phys. Rev, B 41, 10354 (1990).

5. E. Tekman, and S. Ciraci, Phys. Rev. B 43, 7145 (1991).

6. J. A. Nixon, J. H. Davies, and H. U. Baranger, Phys. Rev. B 43, 12638 (1991).

7. Y. B. Levinson, M. I. Lubin, and E. V. Sukhorukov, Phys. Rev. B 45, 11936 (1992).

8. Y. Takagaki, and D. K. Ferry, Phys. Rev. B 46 , 15218 (1992).
9. Ch. Kunze, and R. Lenk, Solid State Commun. 84, 457 (1992).

10. A. Grincwajg, M. Jonson, and R. I. Shekhter, Phys. Rev. B 49, 7557 (1994).

11. Q. Hu, Appl. Phys. Lett. 62, 837 (1993).

12. S. Feng, and Q. Hu, Phys. Rev. B 48, 5354 (1993)

13. R. A. Wyss, C. C. Eugster, J. A. del Alamo, and Q. Hu, Appl. Phys. Lett. 63, 1522 (1993).

14. L. Y. Gorelik, A. Grincwajg, V. Z. Kleiner, R. I. Shekhter, and M Jonson, Phys. Rev. Lett. 73, 2260 (1994).

15. T. J. B. M. Janssen, J. C. Maan, J. Singleton, N. K. Patel, M. Pepper, J. E. F. Frost, D. A. Ritchie, and G. A. C. Jones, J. Phys. Condens. Matler 6,L163 (1994).

16. P. F. Bagwell, and R. K. Lake, Phys. Rev. B 46, $15329(1992)$

17. C. S. Tang, and C. S. Chu, to be published. 
18. The existence of the QBS in a narrow channel, be they impurity-induced or oscillating-barrierinduced, is more a characteristics of the narrow channel than the way they are being induced. Detail discussions have been given by Bagwell and Lake in Ref. 16. In the following, we supplement by presenting a DOS argument. The DOS in a narrow channel is given by $\rho(\vec{x} ; E)=-\operatorname{Im} G^{r}(\vec{x}, \vec{x} ; E) / \pi$, where, in our choice of units, the retarded Green's function $G^{r}\left(\vec{x}, \vec{x}^{\prime} ; E\right)=\sum_{n}\left[\phi_{n}(y) \phi_{n}\left(y^{\prime}\right) /\left(2 i \kappa_{n}\right)\right] e^{i \kappa_{n}\left|x-x^{\prime}\right|}$. Here $\kappa_{n}=\sqrt{E-\varepsilon_{n}}$, and $n$ is the subband index. It is clear from the DOS expression that for $E$ just below $\varepsilon_{N}$, the subband $N$ does not contribute to the DOS. In addition, the wavefunction for this energy $E$ and in this subband $N$ is evanescent along the longitudinal direction. However, if we allow $E$ to be analytically continued into the lower half complex energy plane, such that $E=\varepsilon_{N}-\delta E-i E_{i}$, the contribution of the subband $N$ to the DOS becomes $\left[\phi_{N}^{2}(y) / 2 \pi\right]\left[\delta E^{2}+\right.$ $\left.E_{i}^{2}\right]^{-1 / 4} \sin \left[(1 / 2) \tan ^{-1}\left(E_{i} / \delta E\right)\right]$, which, when $\delta E \sim E_{i} \sim 0$, can be very large. Thus, in the presence of elastic scattering (such as induced by impurities) or inelastic scattering (such as induced by oscillating-barriers, or even photons) events which allow the electrons to make transjtions to the vicinity of, and just below, a subband bottom, the transition can be much enhanced by the large DOS, provided that there is a small and negative imaginary part to the energy. Given the evanescent nature of the state and the small and negative imaginary part in the energy, it is not unexpected that the state can become a QBS.

19. M. Büttiker, Phys. Rev. B 41, 7906 (1990). 